Article

\title{
Shoulder Kinematics Assessment towards Exoskeleton Development
}

\author{
Pablo Delgado ${ }^{1}$, Sajja Alekhya ${ }^{2}$, Amirhossein Majidirad ${ }^{1}$, Nils A. Hakansson ${ }^{2}$ (i), \\ Jaydip Desai ${ }^{2}$ (1) and Yimesker Yihun ${ }^{1, *}$ \\ 1 Mechanical Engineering, Wichita State University, Wichita, KS 67260 , USA; \\ padelgado@shockers.wichita.edu (P.D.); axmajidirad@shockers.wichita.edu (A.M.) \\ 2 Biomedical Engineering, Wichita State University, Wichita, KS 67260, USA; \\ vxsajja@shockers.wichita.edu (S.A.); nils.hakansson@wichita.edu (N.A.H.); jaydip.desai@wichita.edu (J.D.) \\ * Correspondence: yimesker.yihun@wichita.edu
}

Received: 5 August 2020; Accepted: 9 September 2020; Published: 11 September 2020

\begin{abstract}
Neuromuscular and sensorimotor degeneration caused by stroke or any other disease significantly reduce the physical, cognitive, and social well-being across the life span. Mostly, therapeutic interventions are employed in order to restore the lost degrees-of-freedom (DOF) caused by such impairments and automating these therapeutic tasks through exoskeletons/robots is becoming a common practice. However, aligning these robotic devices with the complex anatomical and geometrical motions of the joints is very challenging. At the same time, a good alignment is required in order to establish a better synergy of human-exoskeleton system for an effective intervention procedure. In this paper, a case study of an exoskeleton and shoulder joint alignment were studied through different size and orientation impairment models through motion capture data and musculoskeletal modeling in OpenSim. A preliminary result indicates that shoulder elevation is very sensitive to misalignment and varies with shoulder joint axes orientation; this is partly due to drastic displacement of the upper arm axes with respect to the shoulder joint origin during elevation. Additional study and analysis is required to learn any possible restraint on shoulder elevation that could potentially help in the exoskeleton development.
\end{abstract}

Keywords: shoulder kinematics; rehabilitation; joint alignment; biomechanics; movement analysis

\section{Introduction}

The shoulder is one of the most important and complex human joints due to its wide range of motion. It plays a vital role in the activities-of-daily living in placing and orienting the arm. A limitation in the range of motion ( $\mathrm{ROM})$ of the shoulder can reduce the quality of life [1-3]. The ROM of the shoulder can be affected or reduced by motor impairments, due to strokes, Spinal Cord Injury (SCI), or any physical activity that results in an injury. Therapeutic interventions are usually required for recovering most of the lost ROM. Traditionally during therapy, subjects undergo one-on-one manually assisted exercises with qualified professionals. Depending on how severe the mobility problem of the shoulder is, these sessions can last several months, which ends up being very expensive. Furthermore, manually assisted or conventional therapy lacks training intensity. Verena [4] suggested that an increase in intensity improves rehabilitation with the use of novel therapy methods (e.g., smart robot-based rehabilitation). However, it is difficult to determine the level of muscle activity being triggered in order to maximize the affected limb recovery during therapy sessions. One approach to overcome these limitations is with the use of exoskeletons. These mechanisms can perform more intensive training sessions, measure forces being applied and trajectories being followed, and muscles activities being 
triggered in order to enhance conventional therapy [4]. Nevertheless, these improvements do not solve the issues with exoskeleton alignment and fitting due to complex motion of the shoulder joint.

A lot of effort has been put into studying and modeling the human motion with the hope of building exoskeletons that are capable of mimicking the movement of human limbs (i.e., replicating the workspace). In order to build effective exoskeletons, there has been considerable research directed toward the study and mathematical modeling of human anatomy $[5,6]$. These models coupled with motion capture systems are used in order to design and assess assisted rehabilitation procedures for shoulder impairment and pain due to strokes [7].

This study is aimed at reviewing the shoulder geometry of motion, its modeling and implication towards the design and application of exoskeletons for upper-arm rehabilitation tasks, and to provide a case study of exoskeleton joint impairment. The paper is organized into five related topics: (1) biomechanical models of the shoulder and its complexity; (2) shoulder kinematics and functional assessments; (3) upper arm and shoulder muscle integration and responses during shoulder joint movements; (4) shoulder kinematics and its implication on exoskeleton designs; and (5) best practices and considerations for the design of bio-inspired exoskeletons.

\section{Biomechanical Models of the Shoulder and Its Complexity}

The shoulder also known as glenohumeral joint that articulates between the rounded head of the humerus and the shallow peer-shaped glenoid cavity of the scapula. It is one of the most complex human joints, granting its largest range of motion. As shown in Figure 1, it is composed of mainly three bones named Clavicle, Scapula, and humerus [8,9].The clavicle is a thin s-shaped bone, it is the only connection between the shoulder joint and the body trunk, also known as sternum, at one of its ends forming the Sternoclavicular (ST) joint. On the other end, it links through the Acromioclavicular (AC) joint with the Acromion, a process of the Scapula or, as it is known due to its shape, the shoulder blade. The Humerus, the largest bone of the shoulder, is coupled with the Glenoid Fossa that is contained in the shoulder blade and the humerus head, forming the Glenohumeral (GH) Joint. This union resembles a ball-and-socket joint and 2/3 of the total ROM of the shoulder is attributed to it. The wide ROM is due to the humerus head being to big for the cavity of the glenoid fossa; however, it makes this joint the most unstable of all.

Mathematically modeling the shoulder mechanism is not an easy task, apart from the bony structure, ligaments that attach each bone and muscles are involved in the dynamics and kinematics of it. Consequently, the shoulder bones intercepts each other at different locations, making it a multiple closed-loop chain mechanisms, thus, increasing its complexity. one of the earliest mathematical models of the shoulder complex consisted of 21 muscles and a three rigid body twelve DOF mechanism [10], 3 DOF orientation for each bone, and 3 DOF the displacement of the Humerus head. Another model was presented by Van der helm [11,12], obtaining good results. The model contains four bones, three joints, three extracapsular ligaments, the scapulothoracic gliding plane, and 20 muscles and muscles parts, resulting in a seven DOF mechanism, these models can be used to study the morphological structure of the shoulder. Additionally, mathematical models are used to produce an estimated shoulder workspace that can be later used for exoskeleton design [13-16]. More novel and simplistic models for the kinematics are studied in [6], where IMU sensor data is collected for upper limb kinematic reconstruction, modeling the shoulder as a five DOF mechanism. Furthermore, some researchers neglect the effect of the AC and ST joints, and represent the shoulder kinematics as a pure three-DOF spherical joint [17], assuming the center of rotation of the GH joints is accurately known and fixed.

\section{Shoulder Kinematics and Functional Assessments}

The shoulder has one of the largest ROM of human joints. It allows for people to place and orientate their arm for performing ADL such as grabbing and drinking a glass of water, opening/closing a door, shoelace tying, showering, dressing, self-feeding, and many more. The important functionality of this 
joint can be affected due to an impairment caused by strokes or other diseases. Most of the patients that survive strokes exhibit motor deficits on the shoulder that greatly affect the ROM reducing the accessible workspace [1-3]. Therefore, this will impact the way affected people live, who will probably need assistance from there on to be able to do ADL. Thus, without being able to take care of themselves, shoulder impairment will indeed decrease their quality of life.

Therapeutic treatment plays an important role in restoring the lost ROM in order to overcome such difficulties $[18,19]$. This therapeutic intervention is based on one-on-one training sessions with qualified professionals. During these sessions, therapists assist patients to perform rehabilitation exercises in order to stimulate and trigger muscle activity on the affected limb. Notably, the time frame for a patient to recover most of the lost ROM from an impaired shoulder depends on the severity of the motor deficit, it can take months of sessions with a professional to achieve it. Therefore, the rehabilitation process can end up being very expensive for a patient to cover. On the other hand, it is very difficult to make an assertive diagnosis of the progress and obtain feed backs from the exercise sessions.

As an example, one of the objective of therapeutic sessions is to enhance the muscle strength, however, one should ask how accurate a therapist would be on determine the amount of aid that a patient needs over the session is a challenge. Therefore, the recovery session could not be optimal, since the muscles under treatment are not being triggered adequately. In addition, training sessions are being given by people who will experiment physical fatigue due to repetitiveness and duration of exercises, thus affecting the quality of the exercise.

\subsection{Functional Assessment Tests}

Functional assessment tests are often used to analyze and track the recovery of a patient under rehabilitative exercise routines by physiotherapist. These tests were mainly used in conventional physiotherapy, but, in the recent years, popular functional assessment tests, such as Fugl-Meyer assessment test for motor recovery and modified Ashworth scale made their way into exoskeleton research for validating rehabilitative exoskeleton designs.

The reason why Fugl-Meyer scale and modified Ashworth scale are preferred over the other measurement scales, such as National Institute health stroke scale (NIHSS) [20] and Mayo Portland adaptability Inventory (MPAI-4) [21], is because the task to be done and the scoring system is clear and hence, easily implementable. There are many researches indicating the efficiency of these scales especially in stroke research $[22,23]$.

\subsection{Fugl-Meyer Test}

The Fugl-Meyer test is a score-based test where the maximum possible score is 226 under which the scores attainable for upper extremity range from 0-66. The Fugl-Meyer scale is divided into each part of the body (elbow, wrist, knee, hip, and plantiflexors) and the values are assigned according to severity of the impairment in terms of mild, moderate, and severe [24]. The score is generally assigned by a physiotherapist based on visual assessment and approximation of the patients range of motion and patient feedback.

The Fugl-Meyer test can assess the effectiveness of an exoskeleton by quantifying the Range of motion of a patient over the course of rehabilitative exercises. It is crucial to apply assessment techniques to exoskeleton research because: (1) the outcomes can provide insight on what design parameters are effective in rehabilitation and (2) serve as a basis for standardization of research on exoskeleton design that can help track the developments of design overtime. The score can be determined with the help of a Fugl-Meyer score card that is used by physiotherapists to record the profile of the patient in regards to movement.

For Fugl-Meyer score detection, some researches utilized technology instead of physiotherapist evaluation to reduce the dependence on clinical evaluation, hence making the rehabilitation evaluation process more patient friendly [24]. The technology used to measure Fugl-Meyer source vary from wearable sensor network to depth sensing cameras. 


\subsection{Modified Ashworth Test}

Modified Ashworth scale accounts for the muscle 'spasticity', which translates to muscle pull or stiffness. The modified Ashowrth scale is also a score based system but unlike the Fugl-Meyer scale it measures how stiff or lack there of is the muscle overtime. It consists of six point scores $(0,1,1+, 2,3$, and 4$)$ that amount to the muscle tone with zero being least increase in muscle tone and four is highest muscle tone (rigid) [25].

In the clinical settings, the modified Ashworth test scores are assigned on the basis of physiotherapist analysis and patient feedback, which can drastically vary overtime, hence causing uncertainty in results, which is why a technological method to measure the score would give a standard result for all research in this field and help track the rehabilitation process overtime. The Modified Ashworth scale has been correlated with apparatus, such as goniometer, and also EMG (electromyogram).

\section{Shoulder Kinematics and Rehabilitation}

Motor [26] and muscle responses [27] of the shoulder are needed before, during, and after the rehabilitative process in order to understand the responses of shoulder over the course of rehabilitation. The functional assessment tools are vital to measure the responses, such as Motor response, Fugl-Meyer scale, and Muscle response, Modified Ashworth scale. Understanding such responses during therapy sessions with and without exoskeletons may provide an insight to the variations caused by potential misalignment and other human-exoskeleton interaction factors.

\subsection{Shoulder Kinematics and Motor Response}

The technical measure of Fugl-Meyer score can determine the motor response of the shoulder. The general procedure majority of research have used for determining Fugl-Meyer score is as follows-

IMU sensors are used at the sternum, biceps, and triceps to make a sensor network that can record the position of the Bony landmarks at different instances during various activities. The data are then subjected to feature extraction and then a score is determined in correlation to the Fugl-Meyer score. The RRelief algorithm is widely used for feature extraction because of its better performance as compared to other algorithms [28] and noise tolerance [29]. The RRelief algorithm ranks the attributes according to their ability to maximize the separation among classes that are associated with different clinical scores.

An example of automated model that can measure, analyze, and calculate the scores is presented in [30] which uses the RRelief algorithm for feature extraction and flex sensors alongside accelerometers for additional data. In the quantitative model, the intensity, orientation, and signal complexity are extracted from the raw sensor data and once de-noised the extreme learning machine (ELM) algorithm is adopted to establish the mapping model, which will, in turn, calculate the motor response.

\subsection{Shoulder Kinematics and Muscle Response}

The Electromyogram (EMG) measures the muscle response to a particular stimuli. EMG can be used to see the muscle improvement overtime, since neurological disorders, like stroke, cause muscle stiffness. The modified Ashworth scale can serve as a good tool to quantitatively measure the muscle 'spasticity' and the overtime developments in regards to muscle movement. There are several researches that have correlated EMG data with modified Ashworth scale.

Muscle response of bicep and triceps is much more feasible due to more surface area, which is why the EMG data acquisition methods have mostly been used at these regions, the location of focus for each research may vary, but the principles of the data acquisition and data processing methods and MAS comparison is similar for shoulder. A typical EMG electrode placement ranges from eight electrodes to sixteen electrodes [31]. For instance, in [32], the electrodes are placed on Deltoid, Biceps, Triceps, Flexors, and Extensors muscles. 


\section{Design of Exoskeletons to Align with Shoulder Kinematics}

As the mathematical modeling of the shoulder is very complex, more simplified models are being used for exoskeleton design and alignment. Often, researchers approximate the shoulder mechanism as a pure spherical joint with its center of rotation at the head of the humerus $[17,33,34]$. This type of design assumes the shoulder girdle motion can be neglected, and the center of rotation of the $\mathrm{GH}$ joint is accurately known and fixed over the whole range of motion of the shoulder. However, this is not the case, as studied in [35], where the human joint shifts away its mechanical counterpart. Any misalignment between the exoskeleton and the human shoulder can cause discomfort and produce reaction forces that lead to negative effects over time. Additionally, from the mechanical perspective, spherical joint mechanism is modeled by 3 revolute joints, which include singularity configurations to reduce the exoskeleton performance. Reference [36] proposes a redundant $4 \mathrm{R}$ mechanism in order to avoid singularities; theoretically, this design can operate over the whole workspace of the shoulder, thus making it a simple exoskeleton to model and build. Nonetheless, it does not address the misalignment that was provoked by the shoulder girdle motion or how to accurately locate the center of rotation of the shoulder over the the range of motion of the shoulder. Relatively, a more realistic designs have been proposed and discussed in [37-39], where the position of the humerus head is estimated. These exoskeletons take into account the motion of the humerus head due to shoulder girdle. For example, the MEDARM [37] is a rehabilitation robot with five DOF at the shoulder complex, three DOF for the GH joint, and two DOF for the ST joint to compensate the translation of the humerus head. Notably, the ARMIN III [39] is an exoskeleton that has been used for rehabilitation. In this case, it has been designed to compensate for shoulder girdle motion by approximating the humerus head motion by a circle. However, it assumes that the motion of the humerus head is the same for individuals of the same height. Even though these exoskeletons address the translation that the humerus head suffers during the shoulder motion, they still have to be aligned with their human counterpart, which is very challenging and tedious, and it varies with each individual.

Another challenge for exoskeleton design is the collection of data for the construction of these rehabilitation robotic devices. In particular, one popular approach to collect information about a limb trajectory is with the used of motion capture systems, see [6]. By placing IMU sensors over the skin at specific locations of the limb being studied, it is possible to reconstruct the trajectory and orientation, information that can be used as an input for exoskeleton design algorithm. However, because these sensors are placed on the skin, the measurement will have errors due to the natural motion of the skin over the trajectory being performed. This error will be carried out to the exoskeleton design, which further increases the misalignment between the mechanism and joint understudied. A similar limitations may exist when using MoCap systems as markers that are placed on bony part of the limb are still subject to slight movement, and this small displacement will be transferred to simulation model in OpenSIM, which is discussed in further details in this paper. It is clear that another approach has to be taken in order to produce exoskeletons that can fully reproduce the motion of human joints, especially the shoulder complex range of motion. Reference [40] suggests that probably researchers should embrace redundancy instead of avoiding it as new kinematic representation for exoskeleton design, in order to build mechanism that identically reproduce limbs workspace. In fact, in [41], a novel task-based exoskeleton design is proposed. This approach is focused on the task without mimicking the human joints, therefore avoiding the drawbacks of exoskeleton design that resembles human anatomy, as a matter of fact, an implementation to reproduce a task for a lower limb, the knee, is presented. The resultant mechanism is a one DOF that is able to reproduce the complex three-dimensional (3D) motion of the knee, demonstrating the effectiveness of the approach. 


\section{Exoskeleton Alignment and Its Effect on the Shoulder Muscles: Case Study}

Through an experiment, shoulder movement is studied in a pick-and-place task performed by a person without upper arm movement disability. A $2 \mathrm{lb}$ weight placed on top of a table is used for this purpose as the person is seated behind the table. The trajectory of the right arm is recorded by an integrated motion capture (MoCap) system. The collected data are processed to replicate the same movement in software which provides a better understanding of the mechanism driving the arm and muscles contributing to generate sufficient force as well. In this section, the effect of exoskeleton intervention in upper arm and specifically shoulder rehabilitation is assessed through introducing a certain type of non-anatomical shoulder joint rotation due to interactive forces between exoskeleton and upper arm that could directly affect shoulder joint. Traditionally, exoskeletons are designed to align with the human joint axes of motion by assigning each human joint with an equivalent exoskeleton joint (e.g., a hinge joint for the elbow). This assumes that the location of the axis can be accurately known, and that such a fixed axis exists for the range of motion of the joint or set of joints, which is not always the case. For example, the human shoulder permits complex motions, in which the shoulder joint's center of rotation moves with different arm motions. Misalignment can create large stresses on the attached systems and underlying human anatomy.

Upper limb exoskeletons are modern devices meant to enhance upper-limb functionalities through providing adequate support for people with disabilities in their ADL [42], rehabilitation for patients suffering post-stroke trauma [43], power amplification in an industrial [44] or medical settings [45], or people with neuromuscular injuries [46]. High demands of such profitable machines compelled researchers and the private sector to develop more efficient control systems and mechanism to optimize exoskeletons functionality through intelligent human robot interaction [47]. There are several aspects to investigate the interaction between human and robot counterpart, namely, the design of the mechanism and antropometric characteristics considered in design; actuation of motors and transmissibility of the device while adjusting necessary stiffness or compliance; control type selection [48].

The anatomy of shoulder girdle encompasses several articulations or joints connecting the upper limb to the skeleton. There exist three bones, including scapula (shoulder blade), clavicle, and humerous (upper arm bones) at the shoulder site. Major joints at shoulder girdle are glenohumeral $(\mathrm{GH})$, acromioclavicular (AC), and sternoclavicular (SC) joint (Figure 1). The GH joint is also known as ball and socket joint, which produces a substantial portion of the shoulder movement [49]. While GH is often considered to have three DOF [50], its axis of rotation changes as shoulder moves; hence in simulation, it is crucial to implement a clearance area so as to let GH anatomically moves as human moving his/her arm. There are studies where various methods are applied in order to replicate shoulder movements under different conditions with various level of impairment [51,52]. Arm rotation that originates at the shoulder joint can also be affected through any simplification in modeling, or external pressure from an outside agent, such as exoskeletons. 


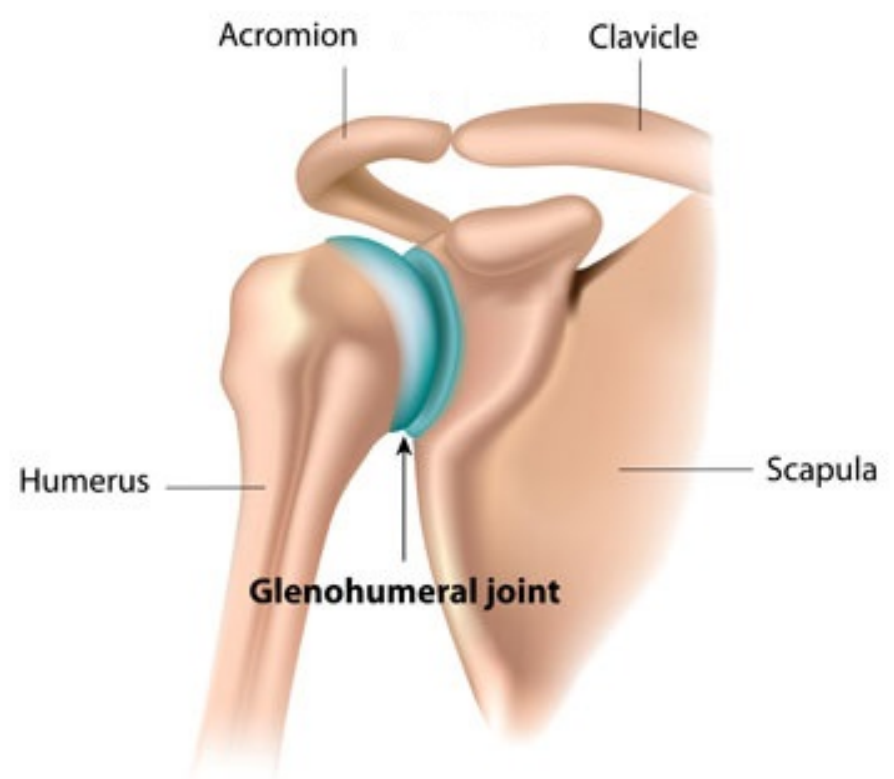

Figure 1. Anatomy of shoulder joint and shoulder girdle.

Robotics has been center of attention in rehabilitation and the way it facilitates training has been tremendously helpful to physicians; however, there are some technical issues involved, which makes equipping controlling such devices challenging at times [53]. Exoskeletons as a popular type of robotic devices in clinical settings may introduce unintended external force or misalignment. In this part, a particular type of shoulder joints misalignment has been investigated through monitoring the muscle compartments at shoulder and specifically those controlling arm rotation through the pick-and-place task.

Picking and placing objects encompasses many activities people typically do, such as opening a sliding window, opening drawer placing clothes, ironing cloth, etc. Accordingly, pick-and-place of a weight has been selected for experiment in order to simulate most of these common tasks. However, the outcome from this study can be extended to a broad range of daily activities people suffering from shoulder impairment might desire to do. Through this study, results will be helpful in assessment of related tasks, namely catching efficiency, grasping assistance, placing efficiency, reaching force, and so on.

\subsection{Methodology}

Research discussed in this section is aimed at obtaining motion capture data, simulation of shoulder movement for a healthy case, as well several other cases where an exoskeleton has imposed unintended pressure to the shoulder joint as the arm performing a certain task. Ten markers are placed at different sites of the fore arm and upper arm in such a way to appropriately capture the motion path of the arm [54,55] as the weight is picked up, moved to right, placed down, and sequence repeats once more to have the weight back to its original location. In addition to necessary sites, additional markers are used to accurately track the upper arm motion during the course of action.

OpenSIM musculoskeletal modeling software is utilized to replicate the arm motion by solving inverse kinematics (IK) equations using designated markers. OpenSIM provides two approaches for calculating the muscle activation levels. The first, Static Optimization (SO), distributes overall joint moments into forces generated by each muscle at any time frame [56]. Basically the movement of 
the model is equipped to solve the equations of motion for the forces under the following conditions (Equation (1)):

$$
\begin{gathered}
\sum_{i=1}^{n_{i}}\left(a_{i} F_{i}^{0}\right) r_{i, k}=\tau_{k i} \\
\sum_{i=1}^{n_{i}}\left[a_{i} f\left(F_{i}^{0}, l_{i}, v_{i}\right)\right] r_{i, k}=\tau_{k c}
\end{gathered}
$$

while minimizing the cost function (Equation (2)).

$$
J=\sum_{i=1}^{n_{i}}\left(a_{i}\right)^{p}
$$

where $n_{i}$ represents the number of muscles consisted in the model, $a_{i}$ denotes the activation level of muscle $i, l_{i}$ denotes the muscle fiber length, $r_{i, k}$ denotes the moment arm of the muscle force about $k$-th joint axis, $v_{i}$ denotes shortening speed of the muscle, $p$ denotes a constant defined by the muscle properties and $f\left(F_{i}^{0}, l_{i}, v_{i}\right)$ represents force-length-velocity criteria, $\tau_{k i}$ denote the torque for ideal force generators, and $\tau_{k c}$ denotes the torque constrained by force-length parameters [57,58]. The second approach to calculate the level of muscle excitation is known as Computed Muscle Control (CMC) [59]. This method calculates muscle activations to drive the system, so that the desired trajectory of the task is best met. First, the desired accelerations are calculated using Equation (3).

$$
\begin{aligned}
\ddot{q}^{*}(t+\Delta t)=\ddot{q}^{\exp }(t+\Delta t) & +k_{v}\left[\dot{q}^{\exp }(t)-\dot{q}(t)\right] \\
& +k_{p}\left[q^{\exp }(t)-q(t)\right]
\end{aligned}
$$

where $q$ denotes the model coordinates, $\ddot{q}^{*}$ denotes the desired acceleration, $q^{\exp }$ denotes the position derived from the experiment, and $k_{v}$ and $k_{p}$ are proportional constants for velocity and positions set for the simulation. With this CMC process, actuator controls that lead to the desired accelerations are computed and, subsequently, a forward dynamics analysis is performed. This cycle iterates until the time reaches the end of the task interval.

For this study, Stanford VA upper limb model comprised of 50 muscles crossing through shoulder, elbow, forearm, and wrist introducing 15 degrees of freedom is used to establish the simulation [60]. Markers pertaining to shoulder were placed at superior, anterior, lateral, and posterior side of that; biceps, medial and lateral elbow, forearm, medial, and lateral wrist are other locations that are selected for marker placement Figure 2. Same protocol has been followed to establish marker registration in OpenSIM and replicate the motion of interest Figure 3. Shoulder joint motion limit is assured by removing all muscles from the model to make sure arm posture is realistic. In addition, muscle wraps (green compartments) are another set of properties the software uses to ascertain muscles are not passing through bones. All of these considerations are embedded in the selected model Figure 4a; however, figures also indicate that all measures are taken into account. 


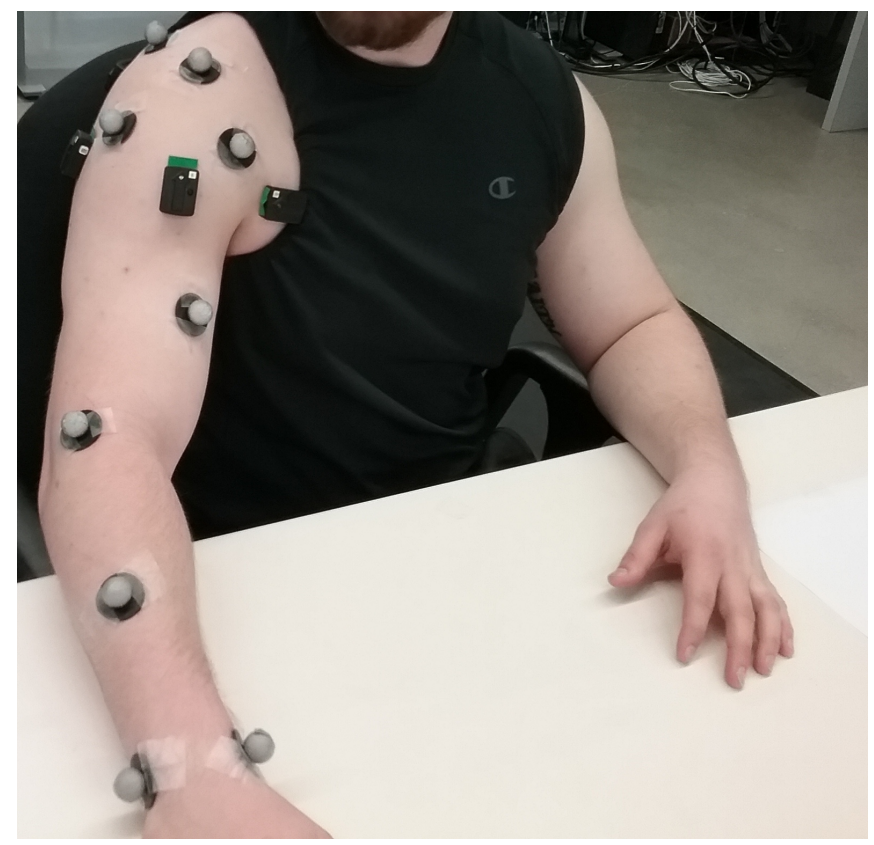

Figure 2. Experimental setup and marker placement.

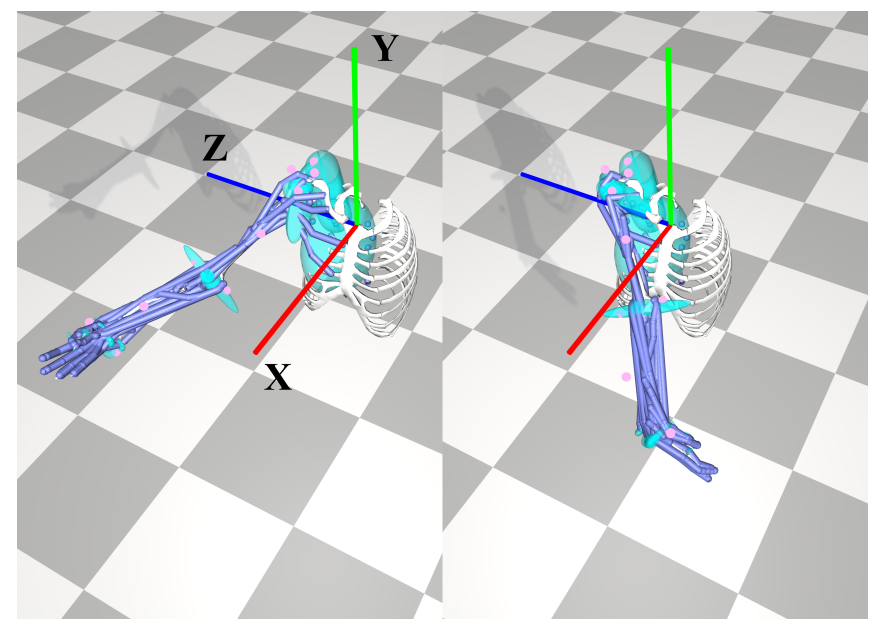

Figure 3. Pick-and-place trajectory in OpenSIM.
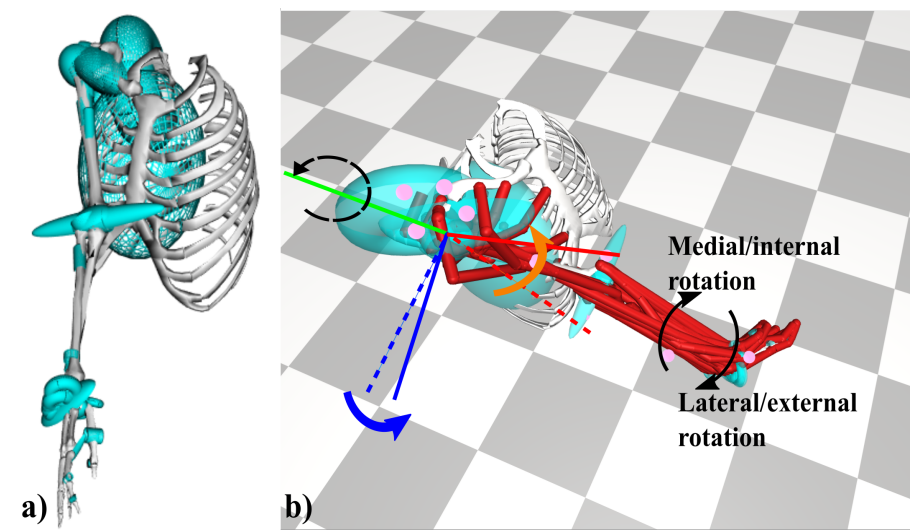

Figure 4. (a) Allowable joint motion limit, (b) Shoulder joint alignment, origin (dashed line) and humerous (solid line) orientations. 
Five cases have been observed, including a healthy case and four non-healthy ones, where shoulder impairment is introduced by imposing a predefined offset in rotation about $Z, Y$, and $X$ axes. Lastly, three subsequent rotations about all axes for an identical degree of rotation are also implemented. These are configurations elected for Case I, Case II (Figure 4b), Case III, and Case IV of this assessment, respectively. Rotation offsets are constrained by shoulder joint range of motion and, based on schematics of the simulation, they do not seem too drastic or unrealistic. Coordinate orientation demonstrated in Figure $4 \mathrm{~b}$ pertains to humerous bone, which has a slight natural deviation from shoulder origin (i.e., humphant 1, holzbaur (2005) model). First, three configurations apply a preset rotation to humerous with respect to the origin to the highest allowable amount anatomically pertinent to the axis they are rotating about.

\subsection{Results and Discussion}

According to anatomy of the shoulder, the origin of posterior fiber in deltoid muscle is at spine of the scapula; and, infraspinatus and teres minor are rotator cuff muscles in charge of arm rotation. As expected, muscle force variations are less susceptible to unintended rotation about $Y$ axis; this is due to the fact that shoulder rotation axis is largely dominated by the $Y$ axis. Case II, which represents unintended rotation about $Y$ axis complies with this argument and data for all three muscles closely follow the one for the healthy case (Figures 5-7).

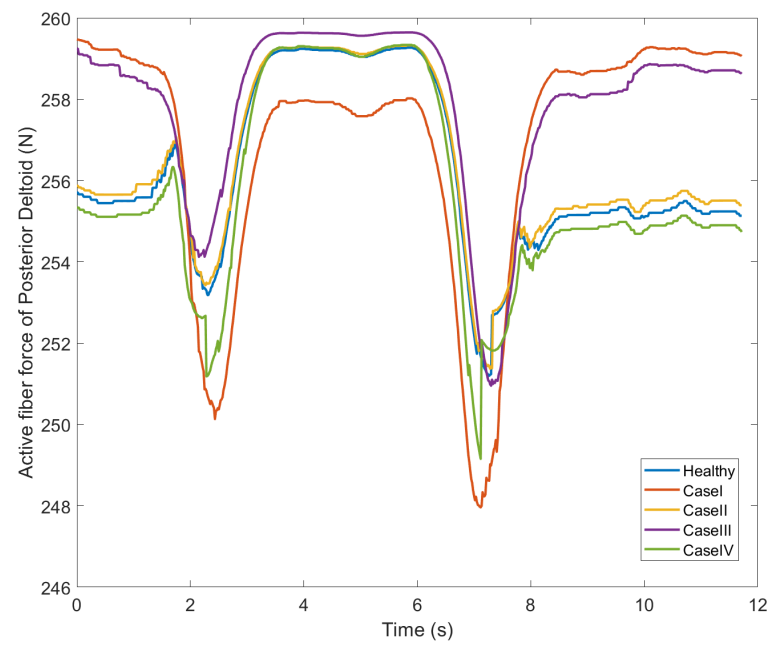

Figure 5. Posterior Deltoid muscle.

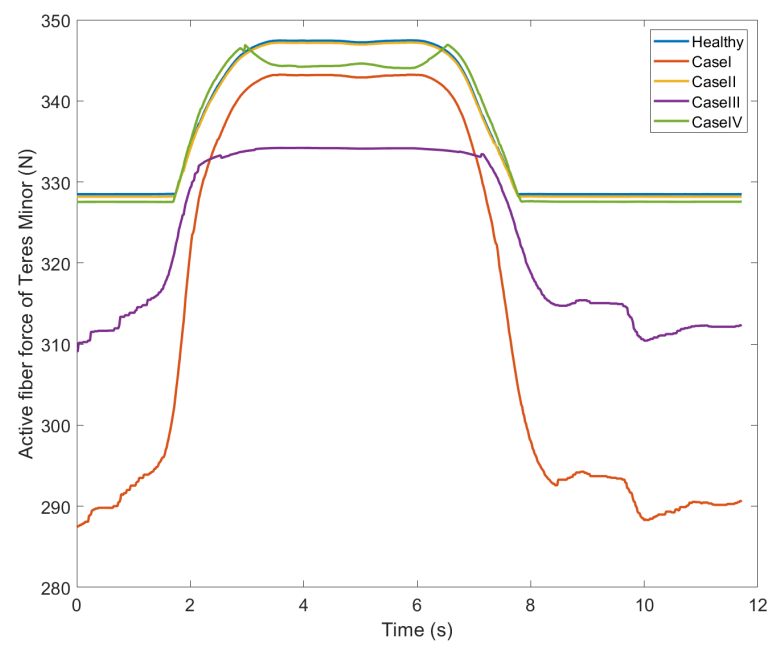

Figure 6. Teres minor muscle. 


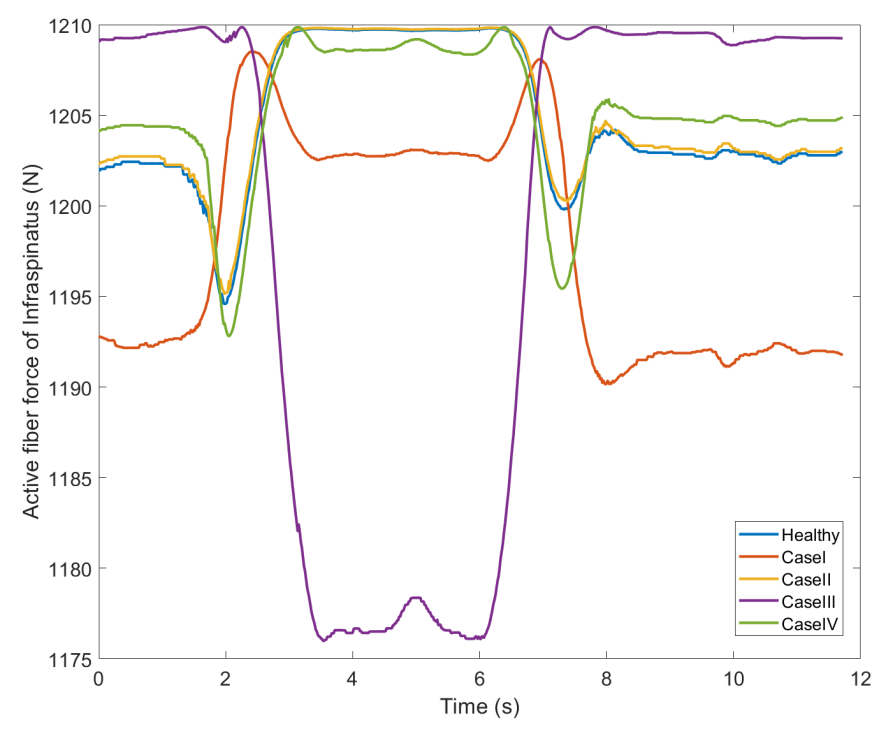

Figure 7. Infraspinatus muscle.

Case IV also indicates some coherence with the healthy case as it consists of subsequent rotations about X, Y, and Z. As for teres minor, all three aforementioned cases show similar pattern and, for other to muscles, they are quite comparable Figure 6. On the other hand as shown in Cases I and III, rotation about $Z$ and $X$ axes are expected to significantly affect active fibers of the pertinent muscles forces, which is demonstrated in muscle force graphs. As for Case I, unintended rotation about $Z$ shows the highest deviation from the anatomically healthy condition.

Rotator cuff muscles show more alike variation of muscle fiber forces when comparing to posterior deltoid, which is expected since the origin and insertion locations are different. Results indicate any misalignment about $X$ and $Z$ axes could cause discomfort and it could lead to irreversible injuries at shoulder joint area in the long term. Evidently, rotator cuff muscles are the most susceptible specifically Infraspinatus (Figure 7) as it shows a drastic drop in muscle force for the swing part of arm movement.

Shoulder elevation is very sensitive to shoulder joint axes orientation and any slight misalignment; this is due to drastic displacement of the upper arm axes with respect to shoulder origin (i.e., humerous and humphant 1, respectively) when elevating (Figure 4b). Evidently, coordinate axes entirely rotate as the shoulder either elevates in frontal plane or moves along the horizontal (transverse) plane. Hence, further evaluation for any possible restraint on shoulder elevation can be performed in future.

\section{Conclusions}

The shoulder's complex anatomy and geometrical motion pose a great challenge for designing assistive technologies, such as exoskeletons. In this study, the results have shown that relatively the shoulder elevation is very sensitive to shoulder joint axes orientation and any slight misalignment; the coordinate axes entirely rotate as shoulder either elevates in frontal plane or moves along the horizontal (transverse) plane. Hence, further evaluation for any possible restraint on shoulder elevation can be performed in the future. Currently, most exoskeleton designs try to mimic the human anatomy, however such approaches are hindered by the fact that the orientation of the axes of the human joints and their location are not accurately known or fixed in the space. For a better synergy of the human-exoskeleton system, analyzing the degree of severity due to motion constraints and alignment of exoskeleton with human joints are required. 
Author Contributions: Conceptualization: Y.Y., N.A.H., J.D., P.D., and A.M.; methodology: P.D., S.A., A.M., Y.Y.; software: P.D. and A.M.; validation: A.M., P.D, Y.Y., J.D. and N.A.H.; formal analysis: P.D., A.M., Y.Y.; investigation: P.D., A.M. and S.A.; resources: Y.Y., N.A.H. and J.D.; data creation: A.M. writing-original draft preparation, P.D., A.S., and A.M.; review and editing: Y.Y., J.D. and N.A.H.; visualization: P.D. and A.M.; supervision: Y.Y., J.D. and N.A.H.; project administration: Y.Y., J.D. and N.A.H.; funding acquisition: Y.Y., J.D. and N.A.H. All authors have read and agreed to the published version of the manuscript.

Funding: This research is funded by National Science Foundation Grant No. 1915872.

Conflicts of Interest: The authors declare no conflict of interest.

\section{References}

1. Chae, J.; Mascarenhas, D.; David, T.Y.; Kirsteins, A.; Elovic, E.P.; Flanagan, S.R.; Harvey, R.L.; Zorowitz, R.D.; Fang, Z.P. Poststroke shoulder pain: Its relationship to motor impairment, activity limitation, and quality of life. Arch. Phys. Med. Rehabil. 2007, 88, 298-301. [CrossRef] [PubMed]

2. Ahlsiö, B.; Britton, M.; Murray, V.; Theorell, T. Disablement and quality of life after stroke. Stroke 1984, 15, 886-890. [CrossRef] [PubMed]

3. Carod-Artal, F.J.; Egido, J.A. Quality of life after stroke: The importance of a good recovery. Cerebrovasc. Dis. 2009, 27, 204-214. [CrossRef] [PubMed]

4. Klamroth-Marganska, V.; Blanco, J.; Campen, K.; Curt, A.; Dietz, V.; Ettlin, T.; Felder, M.; Fellinghauer, B.; Guidali, M.; Kollmar, A.; et al. Three-dimensional, task-specific robot therapy of the arm after stroke: A multicentre, parallel-group randomised trial. Lancet Neurol. 2014, 13, 159-166. [CrossRef]

5. Garner, B.A.; Pandy, M.G. A kinematic model of the upper limb based on the visible human project (vhp) image dataset. Comput. Methods Biomech. Biomed. Eng. 1999, 2, 107-124. [CrossRef]

6. Peppoloni, L.; Filippeschi, A.; Ruffaldi, E.; Avizzano, C.A. A novel 7 degrees of freedom model for upper limb kinematic reconstruction based on wearable sensors. In Proceedings of the 2013 IEEE 11th International Symposium on Intelligent Systems and Informatics (SISY), Subotica, Serbia, 26-28 September 2013; pp. 105-110.

7. Niku, S.B. Introduction to Robotics: Analysis, Control, Applications; John Wiley \& Sons: Hoboken, NJ, USA, 2020.

8. Terry, G.C.; Chopp, T.M. Functional anatomy of the shoulder. J. Athl. Train. 2000, 35, 248.

9. Culham, E.; Peat, M. Functional anatomy of the shoulder complex. J. Orthop. Sports Phys. Ther. 1993, 18, 342-350. [CrossRef]

10. Högfors, C.; Sigholm, G.; Herberts, P. Biomechanical model of the human shoulder-I. Elements. J. Biomech. 1987, 20, 157-166. [CrossRef]

11. Van der Helm, F.C. A finite element musculoskeletal model of the shoulder mechanism. J. Biomech. 1994, 27, 551-569. [CrossRef]

12. Van der Helm, F.C. Analysis of the kinematic and dynamic behavior of the shoulder mechanism. J. Biomech. 1994, 27, 527-550. [CrossRef]

13. Klopčar, N.; Lenarčič, J. Kinematic model for determination of human arm reachable workspace. Meccanica 2005, 40, 203-219. [CrossRef]

14. Klopčar, N.; Tomšič, M.; Lenarčič, J. A kinematic model of the shoulder complex to evaluate the arm-reachable workspace. J. Biomech. 2007, 40, 86-91. [CrossRef] [PubMed]

15. Lau, D.; Eden, J.; Oetomo, D.; Halgamuge, S.K. Musculoskeletal static workspace analysis of the human shoulder as a cable-driven robot. IEEE/ASME Trans. Mechatron. 2014, 20, 978-984. [CrossRef]

16. Kang, B.; Cho, Y.; Cheong, J. Modeling of Human-like Shoulder Complex Robot System. In Proceedings of the 2019 IEEE/ASME International Conference on Advanced Intelligent Mechatronics (AIM), Hong Kong, China, 8-12 July 2019; pp. 857-862.

17. Mustafa, S.K.; Yeo, S.H.; Pham, C.B.; Yang, G.; Lin, W. A biologically-inspired anthropocentric shoulder joint rehabilitator: Workspace analysis \& optimization. In Proceedings of the IEEE International Conference Mechatronics and Automation, Niagara Falls, ON, Canada, 29 July-1 August 2005; Volume 2, pp. 1045-1050.

18. Maulden, S.A.; Gassaway, J.; Horn, S.D.; Smout, R.J.; DeJong, G. Timing of initiation of rehabilitation after stroke. Arch. Phys. Med. Rehabil. 2005, 86, 34-40. [CrossRef] [PubMed] 
19. MajidiRad, A.; Adhikari, V.; Yihun, Y. Assessment of Robot Interventions in a Task-based Rehabilitation: A case study. In Proceedings of the 2018 40th Annual International Conference of the IEEE Engineering in Medicine and Biology Society (EMBC), Honolulu, HI, USA, 18-21 July 2018; pp. 1825-1828.

20. Maas, M.B.; Furie, K.L.; Lev, M.H.; Ay, H.; Singhal, A.B.; Greer, D.M.; Harris, G.J.; Halpern, E.; Koroshetz, W.J.; Smith, W.S. National Institutes of Health Stroke Scale score is poorly predictive of proximal occlusion in acute cerebral ischemia. Stroke 2009, 40, 2988-2993. [CrossRef]

21. Kean, J.; Malec, J.F.; Altman, I.M.; Swick, S. Rasch measurement analysis of the Mayo-Portland Adaptability Inventory (MPAI-4) in a community-based rehabilitation sample. J. Neurotrauma 2011, 28, 745-753. [CrossRef]

22. Gladstone, D.J.; Danells, C.J.; Black, S.E. The Fugl-Meyer assessment of motor recovery after stroke: A critical review of its measurement properties. Neurorehabil. Neural Repair 2002, 16, 232-240. [CrossRef]

23. Platz, T.; Pinkowski, C.; van Wijck, F.; Kim, I.H.; Di Bella, P.; Johnson, G. Reliability and validity of arm function assessment with standardized guidelines for the Fugl-Meyer Test, Action Research Arm Test and Box and Block Test: A multicentre study. Clin. Rehabil. 2005, 19, 404-411. [CrossRef]

24. Del Din, S.; Patel, S.; Cobelli, C.; Bonato, P. Estimating Fugl-Meyer clinical scores in stroke survivors using wearable sensors. In Proceedings of the 2011 Annual International Conference of the IEEE Engineering in Medicine and Biology Society, Boston, MA, USA, 30 August-3 September 2011; pp. 5839-5842.

25. Charalambous, C.P. Interrater reliability of a modified Ashworth scale of muscle spasticity. In Classic Papers in Orthopaedics; Springer: Berlin, Germany, 2014, pp. 415-417.

26. Wiederhold, B. Influence of tracking feedback in user motor response in rehabilitation therapy. Ann. Rev. Cybertherapy Telemed. Adv. Technol. Behav. Soc. Neurosci. 2010, 154, 34.

27. Palazzolo, J.J.; Ferraro, M.; Krebs, H.I.; Lynch, D.; Volpe, B.T.; Hogan, N. Stochastic estimation of arm mechanical impedance during robotic stroke rehabilitation. IEEE Trans. Neural Syst. Rehabil. Eng. 2007, 15, 94-103. [CrossRef]

28. Zebari, R.; Abdulazeez, A.; Zeebaree, D.; Zebari, D.; Saeed, J. A Comprehensive Review of Dimensionality Reduction Techniques for Feature Selection and Feature Extraction. J. Appl. Sci. Technol. Trends 2020, 1, 56-70. [CrossRef]

29. Robnik-Šikonja, M.; Kononenko, I. Theoretical and empirical analysis of ReliefF and RReliefF. Mach. Learn. 2003, 53, 23-69. [CrossRef]

30. Yu, L.; Xiong, D.; Guo, L.; Wang, J. A remote quantitative Fugl-Meyer assessment framework for stroke patients based on wearable sensor networks. Comput. Methods Programs Biomed. 2016, 128, 100-110. [CrossRef] [PubMed]

31. Takala, E.P.; Toivonen, R. Placement of forearm surface EMG electrodes in the assessment of hand loading in manual tasks. Ergonomics 2013, 56, 1159-1166. [CrossRef] [PubMed]

32. Gopura, R.A.R.C.; Kiguchi, K.; Li, Y. SUEFUL-7: A 7DOF upper-limb exoskeleton robot with muscle-model-oriented EMG-based control. In Proceedings of the 2009 IEEE/RSJ International Conference on Intelligent Robots and Systems, St. Louis, MO, USA, 10-15 October 2009; pp. 1126-1131.

33. Garrec, P.; Friconneau, J.P.; Measson, Y.; Perrot, Y. ABLE, an innovative transparent exoskeleton for the upper-limb. In Proceedings of the 2008 IEEE/RSJ International Conference on Intelligent Robots and Systems, Nice, France, 22-26 September 2008; pp. 1483-1488.

34. Naidu, D.; Stopforth, R.; Bright, G.; Davrajh, S. A 7 DOF exoskeleton arm: Shoulder, elbow, wrist and hand mechanism for assistance to upper limb disabled individuals. In Proceedings of the IEEE Africon'11, Livingstone, Zambia, 13-15 September 2011; pp. 1-6.

35. Schiele, A.; Van Der Helm, F.C. Kinematic design to improve ergonomics in human machine interaction. IEEE Trans. Neural Syst. Rehabil. Eng. 2006, 14, 456-469. [CrossRef] [PubMed]

36. Lo, H.S.; Xie, S.S. Optimization of a redundant $4 \mathrm{R}$ robot for a shoulder exoskeleton. In Proceedings of the 2013 IEEE/ASME International Conference on Advanced Intelligent Mechatronics, Wollongong, Australia, 9-12 July 2013; pp. 798-803.

37. Ball, S.J.; Brown, I.E.; Scott, S.H. MEDARM: A rehabilitation robot with 5DOF at the shoulder complex. In Proceedings of the 2007 IEEE/ASME International Conference on Advanced Intelligent Mechatronics, Zurich, Switzerland, 4-7 September 2007; pp. 1-6.

38. Koo, D.; Chang, P.H.; Sohn, M.K.; Shin, J.h. Shoulder mechanism design of an exoskeleton robot for stroke patient rehabilitation. In Proceedings of the 2011 IEEE International Conference on Rehabilitation Robotics, Zurich, Switzerland, 29 June-1 July 2011; pp. 1-6. 
39. Nef, T.; Guidali, M.; Riener, R. ARMin III-arm therapy exoskeleton with an ergonomic shoulder actuation. Appl. Bionics Biomech. 2009, 6, 127-142. [CrossRef]

40. Krishnan, R.; Björsell, N.; Gutierrez-Farewik, E.M.; Smith, C. A survey of human shoulder functional kinematic representations. Med Biol. Eng. Comput. 2019, 57, 339-367. [CrossRef]

41. Yihun, Y.; Adhikari, V.; Majidirad, A.; Desai, J. Task-Based Knee Rehabilitation With Assist-as-Needed Control Strategy and Recovery Tracking System. J. Eng. Sci. Med Diagn. Ther. 2020, 3. [CrossRef]

42. Hall, P.T.; Crouch, D.L. Effect of continuous, mechanically passive, anti-gravity assistance on kinematics and muscle activity during dynamic shoulder elevation. J. Biomech. 2020, 109685. [CrossRef]

43. Franck, J.A. Rehabilitation of Patients With a Moderately to Severely Affected Arm-Hand in the Sub-Acute Phase After Stroke. Ph.D. Thesis, Maastricht University, Maastricht, The Netherlands, 2020.

44. Heo, U.; Kim, S.J.; Kim, J. Backdrivable and Fully-Portable Pneumatic Back Support Exoskeleton for Lifting Assistance. IEEE Robot. Autom. Lett. 2020, 5, 2047-2053. [CrossRef]

45. Jayaraman, A.; Marinov, B.; Singh, Y.; Burt, S.; Rymer, W.Z. Current Evidence for Use of Robotic Exoskeletons in Rehabilitation. In Wearable Robotics; Elsevier: Amsterdam, The Netherlands, 2020; pp. 301-310.

46. Desai, J.; Schabron, B.; Yihun, Y. Force Myography Controlled Intelligent Assistive Wheelchair-Mounted Robotic Exoskeleton for Arm Movements. In Proceedings of the 2019 IEEE International Symposium on Measurement and Control in Robotics (ISMCR), Houston, TX, USA, 19-21 September 2019; pp. D2-D5.

47. Wu, Q.; Wu, H. Development, dynamic modeling, and multi-modal control of a therapeutic exoskeleton for upper limb rehabilitation training. Sensors 2018, 18, 3611. [CrossRef] [PubMed]

48. Gull, M.A.; Bai, S.; Bak, T. A review on design of upper limb exoskeletons. Robotics 2020, 9, 16. [CrossRef]

49. Walden, M. Shoulder Anatomy. 2019. Available online: https://www.sportsinjuryclinic.net/sport-injuries/ shoulder-pain/shoulder-anatomy (accessed on 3 August 2020).

50. Wu, W.; Lee, P.V.; Bryant, A.L.; Galea, M.; Ackland, D.C. Subject-specific musculoskeletal modeling in the evaluation of shoulder muscle and joint function. J. Biomech. 2016, 49, 3626-3634. [CrossRef] [PubMed]

51. De Lima Boarati, E.; Hotta, G.H.; McQuade, K.J.; de Oliveira, A.S. Acute effect of flexible bar exercise on scapulothoracic muscles activation, on isometric shoulder abduction force and proprioception of the shoulder of individuals with and without subacromial pain syndrome. Clin. Biomech. 2020, 72, 77-83. [CrossRef]

52. Whitehair, V.C.; Chae, J.; Hisel, T.; Wilson, R.D. The effect of electrical stimulation on impairment of the painful post-stroke shoulder. Top. Stroke Rehabil. 2019, 26, 544-547. [CrossRef]

53. MajidiRad, A.; Yihun, Y.S. Upper Limb Rehabilitation and Mobility Assistance Using Robotic Devices: A Review. In Proceedings of the ASME 2019 International Design Engineering Technical Conferences and Computers and Information in Engineering Conference, American Society of Mechanical Engineers Digital Collection, Anaheim, CA, USA, 18-21 August 2019.

54. Wu, G.; Van der Helm, F.C.; Veeger, H.D.; Makhsous, M.; Van Roy, P.; Anglin, C.; Nagels, J.; Karduna, A.R.; McQuade, K.; Wang, X.; et al. ISB recommendation on definitions of joint coordinate systems of various joints for the reporting of human joint motion-Part II: Shoulder, elbow, wrist and hand. J. Biomech. 2005, 38, 981-992. [CrossRef]

55. Rab, G.; Petuskey, K.; Bagley, A. A method for determination of upper extremity kinematics. Gait Posture 2002, 15, 113-119. [CrossRef]

56. Anderson, F.; Guendelman, E.; Habib, A.; Hamner, S.; Holzbaur, K.; John, C.T.; Ku, J.; Liu, M.; Loan, P.; Reinbolt, J.; et al. OpenSIM User's Guide; Release 2.4. 2011. Available online: https:/ / simtk-confluence. stanford.edu:8443/display/OpenSim/User (accessed on 3 August 2020).

57. Ackermann, M. Dynamics and Energetics of Walking With Prostheses. Ph.D. Thesis, University of Stuttgart, Stuttgart, Germany, 2007.

58. Ou, Y. An Analysis of Optimization Methods for Identifying Muscle Forces in Human Gait; VDI-Verlag: Düsseldorf, Germany, 2013. 
59. MajidiRad, A.; Yihun, Y.; Desai, J.; Hakansson, N.A. Simulation of Exoskeleton Alignment and its Effect on the Knee Extensor and Flexor Muscles. In Proceedings of the 2019 41st Annual International Conference of the IEEE Engineering in Medicine and Biology Society (EMBC), Berlin, Germany, 23-27 July 2019; pp. 4093-4096.

60. Holzbaur, K.R.; Murray, W.M.; Delp, S.L. A model of the upper extremity for simulating musculoskeletal surgery and analyzing neuromuscular control. Ann. Biomed. Eng. 2005, 33, 829-840. [CrossRef]

(C) 2020 by the authors. Licensee MDPI, Basel, Switzerland. This article is an open access article distributed under the terms and conditions of the Creative Commons Attribution (CC BY) license (http:/ / creativecommons.org/licenses/by/4.0/). 